\title{
Conductas Delictivas Y Antisociales En Adolescentes Que Estudian Y No Estudian
}

\author{
Angélica Romero Palencia, Doctora \\ Araceli Sánchez Solís, Licenciada \\ Arturo del Castillo Arreola, Doctor
}

Universidad Autónoma del Estado de Hidalgo, Instituto de Ciencias de la Salud, Área Académica de Psicología

\begin{abstract}
Adolescence is a stage of transition from childhood to the adult world, which is affected among other things by the tension between the incorporation into the labor market and permanence in the educational system (D'alessandre, 2010). However, there is little information related to adolescents who do not study or work. Because of the information that antisocial and criminal behavior tends to accentuate at this stage of life (Gaeta \& Galvanoski, 2011), this study identifies differences in the presence of antisocial and criminal behavior among students in two public high schools at Pachuca Hidalgo, Mexico, and adolescents who do not study or work in the same city using the AD measure (Seisdedos \& Sánchez, 2001). The sample consisted of 120 teenagers between 14 and 18 years of age, with a total of 81 students and 39 adolescents who do not study or work. Through an analysis of variance ANOVA of sex by occupation were found significant differences in the presence of antisocial behaviors between adolescents who study and those who do not. A difference was observed in the presence of criminal behavior also, specifically in the group of male adolescents who did not study. It was observed that those adolescent men and women who are studying, are less likely to commit criminal acts, compared to those adolescent especially men who are not within a school or work system. The role played by the socio-cultural context as a risk factor or protection for the appearance of criminal and antisocial behavior is highlighted.
\end{abstract}

Keywords: Antisocial behavior, criminal behavior, adolescents, risk factors

\section{Resumen}

La adolescencia es una etapa de tránsito de la niñez al mundo adulto, que se ve afectada entre otras cosas, por la tensión entre la incorporación al 
mercado laboral y la permanencia en el sistema educativo (D’alessandre, 2010). No obstante, es poca la información relacionada con adolescentes que no estudian ni trabajan. Debido a que se tiene información de que los comportamientos antisociales y delictivos suelen acentuarse en esta etapa de vida (Gaeta \& Galvanoski, 2011), el presente estudio tuvo como objetivo identificar las diferencias en conductas antisociales y delictivas en estudiantes de dos escuelas preparatorias públicas del estado de Hidalgo en México y adolescentes que no estudian ni trabajan, en la Ciudad de Pachuca Hidalgo, México, mediante el Instrumento A-D (Seisdedos \& Sánchez, 2001). Participaron 120 adolescentes de entre 14 y 18 años, con un total de 81 estudiantes y 39 adolescentes que no estudian ni trabajan. A través de un análisis de varianza ANOVA de sexo por ocupación se encontraron diferencias significativas en la presencia de conductas antisociales entre adolescentes que estudian y los que no. Se observó una diferencia en la presencia de conductas delictivas, específicamente en el grupo de adolescentes varones que no estudian. Se observó que aquellos adolescentes hombres y mujeres, que se encuentran estudiando son menos propensos a cometer actos delictivos. Se resalta el papel que juega el contexto sociocultural como factor de riesgo o protección para la aparición de comportamientos delictivos y antisociales.

Palabras-clave: Conducta antisocial, conducta delictiva, adolescentes, factores de riesgo

\section{Introducción}

Para Coon \& Mitterer (2011) los problemas en la etapa adolescente se derivan de las normas poco claras sobre el papel que se debe desempeñar, el saber si son adultos o niños, si deben ser autónomos o dependientes, o si deben trabajar o jugar, conlleva a importantes ambigüedades en donde se hace difícil formar imágenes claras de sí mismos y de cómo actuar. Esta postura se complementa con la de D'alessandre (2010) que afirma que el tránsito de la adolescencia al mundo adulto, es afectado por la tensión entre la incorporación al mercado laboral y la permanencia en el sistema educativo.

Por lo que cabe resaltar la situación social en la que los adolescentes en México se encuentran, según el Instituto Nacional de Estadística y Geografía (INEGI, 2014) la tasa de desempleo de 15 a 19 años y de 20 a 24 años son de 9.8 y 9.2\%, respectivamente. Se habla de la existencia de un grupo doblemente excluido que no se identifican con el ser estudiantes o trabajadores estando fuera de la oportunidad de participar del entramado social. 
Cifuentes y Londoño (2011) realizaron un estudio en Colombia, en el que pretendían obtener el perfil cognitivo de reclusos diagnosticados con TPA (trastorno de la personalidad antisocial) y reclusos sin un diagnóstico de tal trastorno, encontraron que la única diferencia en el perfil cognitivo era el grado de educación de los participantes, puesto que, aquellos reclusos diagnosticados en su mayoría sólo habían terminado la primaria, mientras que los reclusos sin el diagnostico poseían una escolaridad mínima de bachillerato. El Centro de Investigación para el Desarrollo [CIDAC], (2015) menciona que en 2012 se procesaron en México, 19,178 adolescentes, de los cuales 405 no sabían leer ni escribir, 634 no habían asistido nunca a la escuela, 2617 estudiaron sólo la primaria, 5990 estuvieron inscritos en la secundaria, 2,647 entraron en la preparatoria y 7290 no pudieron especificar hasta qué grado habían estudiado.

Gaeta y Galvanovskis (2011) en un estudio acerca de la propensión hacia conductas antisociales y delictivas en estudiantes de Puebla, México, de entre 12 y 20 años de edad en el que participaron 150 estudiantes, separados en subgrupos de sexo, edad y estructura familiar, detectaron diferencias estadísticamente significativas entre los adolescentes hombres quienes presentaron mayor propensión a cometer conductas antisociales y delictivas y cometer conductas antisociales agresivas con violencia, que las adolescentes mujeres.

Poca es la información obtenida relacionada a profundidad con las conductas delictivas con adolescentes que no estudian ni trabajan, al dar por hecho que los "delincuentes" no realizan ninguna de estas actividades; sin embargo, en las últimas décadas se han incrementado considerablemente las investigaciones sobre violencia escolar, la cual incluye varios tipos de conductas transgresoras que tienen lugar en escuelas e institutos; desde actos delictivos leves hasta patrones de comportamiento más graves (Cava, Musitu, Buelga, \& Murgui, 2010; Romero, Plata, Domínguez, Martínez, Del Castillo \& Gil, 2014; Plata, Romero, Del Castillo, Domínguez \& Martínez, 2015; López, Romero, \& López, 2016).

Aisladamente los factores de riesgo en las conductas antisociales y delictivas no determinan la existencia de las mismas, sino más bien el conjunto y la interacción entre ellos. De la Peña (2010) propone una clasificación dividiéndola en externos e internos. Los factores externos son aquellos elementos que se encuentran fuera del sujeto pero que interactúan con él, tales como los medios masivos de comunicación, la familia, la situación social, los recursos económicos, etc. Mientras que los factores internos son aquellos elementos que se encuentran dentro del sujeto, relacionados a factores de riesgo, como los factores psicológicos -la impulsividad, la empatía, etc.- y el consumo de sustancias. A continuación se explicarán con mayor detalle. 
Medios de comunicación masivos: su influencia afecta en la presencia de cierto tipo de conductas que pretenden reproducir modelos simbólicos de vida, en ocasiones inalcanzables, llenos de expectativas de una cultura de consumo, e importancia a aspectos materiales (Gutiérrez, Torres, \& Fernanda, 2011).

Familia: el ambiente familiar es el que puede alentar o ayudar a disminuir las conductas antisociales, y se sabe que un ambiente familiar desfavorable se relaciona a la aparición de conductas antisociales (Gaeta \& Galvanovskis, 2011).

Situación social: las teorías ecológicas parten de la idea de que la ciudad "produce" delincuencia. Existen zonas en donde se concentran conductas antisociales y delictivas, favorecidas por la desorganización y el fácil contagio de este tipo de conductas (De la Peña, 2010).

Para Peres (2008) la situación de mayor riesgo en la que se encuentra el adolescente es la falta de recursos económicos, ya que afecta en las demandas de las necesidades para su autorrealización personal, poniendo en conflicto su interacción con el medio ambiente. Con respecto a lo anterior, en Hidalgo actualmente se estima que $50.8 \%$ de la población viven en pobreza, que forman parte del 49\% estimado para el país según la Encuesta Nacional de Salud y Nutrición [ENSANUT], (Gutiérrez, Rivera-Dommarco, ShamahLevy, Villalpando-Hernández, Franco, Cuevas-Nasu, et al., 2012). Igualmente se identifica que $95.2 \%$ de los niños de 6 a 14 años asiste a la escuela, y de los individuos de 15 a 24 años solo el 39.8\%.

Factores psicológicos: hacen referencia a características de la personalidad, problemas de conducta o psicopatológicos y la diferencia de los estilos de afrontamiento (De la Peña, 2010).

Impulsividad: se considera como un elemento predictor de conductas violentas. Se relaciona con la contención de la conducta, y el manejo de emociones (sexuales y agresivas), es un procesamiento rápido de la información (Varela, 2011).

La Empatía: en un análisis llevado a cabo Garaigordobil, Aliri, Martínez, Maganto, Bernaras, y Jaureguizar (2013), encontraron que la empatía es la base de la conducta altruista, resulta incompatible con la aceptación de la agresión, es un instrumento inhibidor de la agresión.

Autoestima: Del Barrio y Roa (2006) exponen que cuando la autoimagen es negativa se presenta una frustración profunda, el resentimiento es una de las fuentes primarias de agresión. La sobrevaloración de sí mismo puede provocar el mismo efecto puesto que se producen percepciones narcisistas que dificultan una buena integración y el rechazo del grupo.

Consumo de sustancias: en un estudio sobre la relación en el consumo de drogas y la presencia de conductas infractoras se muestra que la 
mayoría de los menores que han infringido las normas consumen alguna sustancia (Martínez, Banqueri, \& Lozano, 2012).

Para Marchiori (2011) la personalidad dependiente se relaciona a inestabilidad emocional, social y familiar. La conducta delictiva es una rebeldía en donde existe hiperactividad, irritabilidad, violencia, impulsividad, valores distorsionados que impiden discernir entre aspectos negativos y favorables, justificación de la conducta, disgregación del pensamiento e inestabilidad afectiva.

Por lo anterior se hipotetiza que el afrontamiento a los factores de riesgo constituye un recurso para una adaptación social, evitando conductas antisociales y delictivas, en donde se encuentre en una situación que evite estos factores y que el estar en la escuela sirve como un factor protector para adoptar menos conductas antisociales y delictivas.

El Fondo Internacional de Emergencia de las Naciones Unidas para la Infancia con sus siglas en ingles UNICEF (2012) indica que al acceder a la educación media superior se generan procesos de aceptación o rechazo, interviniendo en el incremento, aparición, eliminación y/o modificación de diversas conductas. La Secretaria de Educación Pública (SEP, 2010) informa que con respecto al nivel educativo medio superior ingresa a primer grado el 96.7\% de los egresados de educación secundaria (aproximadamente 4.2 millones) que es igual al $66.7 \%$ con respecto a la población total de adolescentes de 16 a 18 años de edad. Es decir que un 33.3\% de esta población, (aproximadamente 2.1 millones) no pertenece a ningún sistema educativo.

De acuerdo con el Instituto Mexicano de la Juventud (IMJUVE, 2010) las conductas antisociales y delictivas en adolescentes en Hidalgo; incrementaron en los últimos años, no sólo en adolescentes que no estudian, sino también en los que sí lo hacen.

De ahí que el objetivo del trabajo sea comparar las conductas antisociales y delictivas prevalentes en adolescentes hombres y mujeres de entre 14 y 18 años de edad de la ciudad de Pachuca Hidalgo, México, en una muestra dividida en estudiantes y no estudiantes.

\section{Método}

Participantes: La muestra fue de 120 participantes de entre 14 y 18 años de edad, con un total de 81 estudiantes de Preparatorias Públicas de Pachuca, 41 eran estudiantes de una preparatoria ubicada en el centro de la ciudad, 40 eran estudiantes de una preparatoria ubicada en los límites de la ciudad y 39 adolescentes que no estudian ni trabajan de la ciudad de Pachuca, Hidalgo, con una media de edad de 16.19 años y una D.E. de 0.886. El $43 \%$ eran mujeres y el 57\% hombres. La muestra se seleccionó mediante un muestreo no probabilístico accidental. 
Instrumento: Cuestionario A-D (Seisdedos \& Sánchez, 2001) de preguntas dicotómicas, 20 asociadas a conductas antisociales y 20 a delictiva, con formato único para hombres y mujeres. Con una confiabilidad en la sub escala de conductas antisociales de .866 para hombres y de .860 para mujeres; con respecto a la sub escala de conductas delictivas tiene .862 y .860 para hombres y mujeres respectivamente.

Procedimiento: Se solicitó a los directivos de las instituciones el permiso para aplicar la prueba, posteriormente se acudió al lugar para aplicar el cuestionario. Para cubrir la cuota de los adolescentes que no estudian se acudió a las diversas calles y plazas públicas de la ciudad de Pachuca Hidalgo, México. Al finalizar la participación de estos adolescentes se empleaba la técnica de bola de nieve para completar la muestra. Se sugería omitir su nombre, para tratar de asegurar la honestidad en sus respuestas, no así la edad ni el sexo y se les aseguraba confiabilidad.

\section{Resultados}

Primeramente se presentan las tablas de percentiles de conducta antisocial y delictiva de la muestra, cabe señalar, que los percentiles que aquí se presentan van de acuerdo con los establecidos por Seisdedos y Sánchez (2001) para población mexicana.

Como puede observarse en la Tabla 1, el valor de moda de la muestra en general se encuentra en el percentil 90 y el $43.3 \%$ de la muestra está dentro o por encima del percentil 70. Lo anterior indica que las conductas antisociales están presentes en la mayoría de ellos, llevando a cabo actos como alborotar alguna reunión, ensuciar las calles, molestar o engañar a personas desconocidas, hacer trampas en exámenes, competencias o información de resultados, hacer grafitis, romper cosas de otra persona, comer cuando está prohibido, etc.

Otro dato destacable es que de manera general, pareciera que en la muestra hubo pocas mujeres que mostraron bajos niveles de conductas antisociales y muchas que tienen altos niveles de estas conductas, por lo que la mayoría de la distribución femenil se concentra en los percentiles más altos $(<70)$, mientras que en los hombres se observa una distribución mucho más uniforme de conductas antisociales, con distribución en prácticamente todos los percentiles (Ver Tabla 1). 
Tabla 1. Percentiles de la conducta antisocial en la muestra

\begin{tabular}{|c|c|c|c|c|c|c|c|c|c|c|c|c|}
\hline \multirow[b]{2}{*}{ Percentil } & \multicolumn{2}{|c|}{ General } & \multicolumn{2}{|c|}{ Hombres } & \multicolumn{2}{|c|}{ Mujeres } & \multicolumn{2}{|c|}{$\begin{array}{l}\text { Preparatoria } \\
\text { No. } 3\end{array}$} & \multicolumn{2}{|c|}{$\begin{array}{l}\text { Preparatoria } \\
\text { No. } 4\end{array}$} & \multicolumn{2}{|c|}{$\begin{array}{c}\text { No } \\
\text { estudian }\end{array}$} \\
\hline & $\mathrm{f}$ & $\%$ & $\mathrm{f}$ & $\%$ & $\mathrm{f}$ & $\%$ & $\mathrm{f}$ & $\%$ & $\mathrm{f}$ & $\%$ & $\mathrm{f}$ & $\%$ \\
\hline 3 & 2 & 1.7 & 2 & 2.9 & 0 & 0 & 2 & 4.9 & 0 & 0 & 0 & 0 \\
\hline 5 & 6 & 5 & 6 & 8.7 & 0 & 0 & 3 & 7.3 & 3 & 7.5 & 0 & 0 \\
\hline 10 & 6 & 5 & 6 & 8.7 & 0 & 0 & 3 & 7.3 & 1 & 2.5 & 2 & 5.1 \\
\hline 20 & 15 & 12.5 & 8 & 11.6 & 7 & 13.7 & 6 & 14.6 & 9 & 22.5 & 0 & 0 \\
\hline 30 & 17 & 14.2 & 12 & 17.4 & 5 & 9.8 & 6 & 14.6 & 9 & 22.5 & 2 & 5.1 \\
\hline 40 & 6 & 5 & 3 & 4.3 & 3 & 5.9 & 2 & 4.9 & 3 & 7.5 & 1 & 2.6 \\
\hline 50 & 12 & 10 & 6 & 8.7 & 6 & 11.8 & 5 & 12.2 & 2 & 5.0 & 5 & 12.8 \\
\hline 60 & 4 & 3.3 & 3 & 4.3 & 1 & 2 & 1 & 2.4 & 1 & 2.5 & 2 & 5.1 \\
\hline 70 & 17 & 14.2 & 5 & 7.2 & 12 & 23.5 & 6 & 14.6 & 6 & 15.0 & 5 & 12.8 \\
\hline 80 & 7 & 5.8 & 3 & 4.3 & 4 & 7.8 & 2 & 4.9 & 3 & 7.5 & 2 & 5.1 \\
\hline 90 & 19 & 15.8 & 11 & 15.9 & 8 & 15.7 & 5 & 12.2 & 2 & 5.0 & 12 & 30.8 \\
\hline 95 & 2 & 1.7 & 0 & 0 & 2 & 3.9 & 0 & 0 & 1 & 2.5 & 1 & 2.6 \\
\hline 97 & 6 & 5 & 4 & 5.8 & 2 & 3.9 & 0 & 0 & 0 & 0 & 6 & 15.4 \\
\hline 99 & 1 & 0.8 & 0 & 0 & 1 & 2 & 0 & 0 & 0 & 0 & 1 & 2.6 \\
\hline Total & 120 & 100 & 69 & 100 & 51 & 100 & 41 & 100 & 40 & 100 & 39 & 100 \\
\hline
\end{tabular}

Con respecto a las conductas delictivas existe una diferencia de aproximadamente de 4.5 puntos porcentuales entre el grupo que obtuvo el mayor puntaje (hombres no estudian) y el de menor puntuación (mujeres de la Preparatoria $\left.\mathrm{N}^{\circ} 3\right)$.

Tabla 2. Percentiles de la conducta delictiva en la muestra

\begin{tabular}{ccccccccccccc}
\hline & \multirow{4}{*}{ General } & \multicolumn{2}{c}{ Hombres } & \multicolumn{2}{c}{ Mujeres } & \multicolumn{2}{c}{$\begin{array}{c}\text { Preparatoria } \\
\text { No. 3 }\end{array}$} & \multicolumn{2}{c}{$\begin{array}{c}\text { Preparatoria } \\
\text { No. 4 }\end{array}$} & \multicolumn{2}{c}{$\begin{array}{c}\text { No } \\
\text { estudian }\end{array}$} \\
\hline Percentil & $\mathrm{f}$ & $\%$ & $\mathrm{f}$ & $\%$ & $\mathrm{f}$ & $\%$ & $\mathrm{f}$ & $\%$ & $\mathrm{f}$ & $\%$ & $\mathrm{f}$ & $\%$ \\
\hline 3 & 0 & 0 & 0 & 0 & 0 & 0 & 0 & 0.0 & 0 & 0.0 & 0 & 0.0 \\
5 & 0 & 0 & 0 & 0 & 0 & 0 & 0 & 0.0 & 0 & 0.0 & 0 & 0.0 \\
10 & 0 & 0 & 0 & 0 & 0 & 0 & 0 & 0.0 & 0 & 0.0 & 0 & 0.0 \\
20 & 24 & 20 & 24 & 34.8 & 0 & 0 & 8 & 19.5 & 14 & 35.0 & 2 & 5.1 \\
30 & 0 & 0 & 0 & 0 & 0 & 0 & 0 & 0.0 & 0 & 0.0 & 0 & 0.0 \\
40 & 11 & 9.2 & 11 & 15.9 & 0 & 0 & 5 & 12.2 & 4 & 10.0 & 2 & 5.1 \\
50 & 5 & 4.2 & 5 & 7.2 & 0 & 0 & 3 & 7.3 & 2 & 5.0 & 0 & 0.0 \\
60 & 28 & 23.3 & 3 & 4.3 & 25 & 49 & 8 & 19.5 & 14 & 35.0 & 6 & 15.4 \\
70 & 6 & 5 & 6 & 8.7 & 0 & 0 & 3 & 7.3 & 0 & 0.0 & 3 & 7.7 \\
80 & 19 & 15.8 & 3 & 4.3 & 16 & 31.4 & 11 & 26.8 & 4 & 10.0 & 4 & 10.3 \\
90 & 5 & 4.2 & 4 & 5.8 & 1 & 2 & 0 & 0.0 & 0 & 0.0 & 5 & 12.8 \\
95 & 12 & 10 & 6 & 8.7 & 6 & 11.8 & 2 & 4.9 & 2 & 5.0 & 8 & 20.5 \\
97 & 4 & 3.3 & 4 & 5.8 & 0 & 0 & 1 & 2.4 & 0 & 0.0 & 3 & 7.7 \\
99 & 6 & 5 & 3 & 4.3 & 3 & 5.9 & 0 & 0.0 & 0 & 0.0 & 6 & 15.4 \\
Total & 120 & 100 & 69 & 100 & 51 & 100 & 41 & 100 & 40 & 100 & 39 & 100 \\
\hline
\end{tabular}

Con base en estos resultados hasta aquí expuestos no se puede saber si existe o no una diferencia estadísticamente significativa entre los seis grupos, por lo que para determinar si existen o no, se realizó un análisis factorial de varianza (ANOVA) de dos vías: sexo (hombre y mujer) por ocupación (estudiantes de las dos preparatorias encuestadas y no estudiantes) para encontrar diferencias significativas tanto en el sexo de los participantes, 
como en su ocupación y en la interacción de estas dos variables, con respecto a las escalas de Conductas Antisociales y Conductas Delictivas.

\section{Subescala de Conducta Antisocial}

Se detectaron efectos principales en la ocupación del adolescente $\mathrm{F}$ $(2,114)=17.821 \mathrm{p}=.000$, de acuerdo a la prueba Post Hoc de Tuckey se indica que el grupo de adolescentes que hace la diferencia se significativa es el grupo de adolescentes que no estudian, se detecta que los estudiantes de la preparatoria $N^{\circ} 3(\mu=7.5122)$ y los estudiantes de la Preparatoria $N^{\circ} 4(\mu=$ 7.5500) poseen menos Conductas Antisociales que aquellos adolescentes que no estudian $(\mu=12.6154)$. El ANOVA no mostró diferencia estadísticamente significativa por sexo $\mathrm{F}(1,114)=.002 \mathrm{p}=.966 \mathrm{ni}$ en la interacción de sexo por ocupación. $\mathrm{F}(2,1815.714)=2.493 \mathrm{p}=.087$.

\section{Subescala de Conducta Delictiva}

Se encontró una interacción estadísticamente significativa de sexo por ocupación $\mathrm{F}(2,114)=5.41 \mathrm{p}=0.006$. La prueba Post hoc de Tukey mostró que los hombres que no estudian son significativamente más altos en conducta delictiva $(\mu=8.125)$ que los otros cinco grupos de adolescentes, que son los hombres de la Preparatoria $N^{\circ} 3(\mu=2.500)$, los hombres de la Preparatoria $N^{\circ} 4(\mu=.857)$, las mujeres de la Preparatoria $N^{\circ} 3(\mu=.765)$, las mujeres de la Preparatoria $4(\mu=.368)$, y las mujeres que no estudian $(\mu=$ 3.000).

\section{Conclusión}

Los resultados de esta investigación, muestran que dos de los factores que favorecen la presencia de conductas antisociales y delictivas son el sexo (si es hombre o mujer) y su situación social escolar o laboral en la que se encuentre el o la adolescente, es decir, si se encuentra estudiando y/o trabajando o si no lo hace.

Cabe señalar que aquellos adolescentes tanto hombres como mujeres, que se encuentran estudiando son menos propensos a cometer actos antisociales, en comparación con aquellos hombres y mujeres que no se encuentran dentro de un sistema escolarizado o laboral. Sin embargo se resalta que ninguno de los grupos a los que se les aplicó el cuestionario, se exime de tener conductas antisociales sean hombres o mujeres, estudien o no.

Como supone esta investigación en el caso de aquellos adolescentes que no están bajo algún programa escolar no obtienen procesos de socialización adecuados para comportarse ante el medio ambiente que les rodea, puesto que las normas que se comparten en una escuela, así como los 
distintos tipos de comportamiento, no son igualmente aprendidos por aquellos que no están bajo un régimen educativo.

Flores (2010) en su investigación señala al contexto social (la escuela) como transmisor de valores. Al exponer a un nuevo contexto social a cada uno de los integrantes; diferentes personalidades son encontradas y nuevos valores se interiorizan positiva o negativamente. Escuchar nuevas opiniones genera cierta tolerancia e inclusive empatía entre pares, generando mayores habilidades de afrontamiento ante problemáticas comunes. Se considera a la situación escolar como un elemento influyente en la condición de cada uno de ellos, indicando así su inclusión o exclusión del entramado social, siendo parte fundamental en su afrontamiento al mundo y las conductas que desarrollarán en su vida cotidiana (D’alessandre, 2010).

En un estudio similar (Pérez, Gázquez, Mercader, Molero \& García, 2011) se demostró que el fracaso en el rendimiento académico se asocia con la presencia de conductas antisociales y delictivas; en donde la mayor presencia de ambas se presentaba cuando el estudiante había suspendido alguna vez su preparación académica, y en aquellos alumnos que solamente habían repetido curso marcaban una diferencia sólo en conductas delictivas.

La escuela actúa como agente de socialización donde se adquieren y desarrollan comportamientos de relación interpersonal, aprendiendo normas y reglas que la sociedad ha establecido a través de la interacción con los iguales. Las conductas sociales positivas y negativas conforman un patrón de comportamiento que se adquiere para mantener una relación con el entorno. El asistir a la escuela constituye un entorno relevante en el desenvolvimiento social que, ayuda a enseñar y desarrollar habilidades de relación (Rivas, 2012)

La investigación presente indica que específicamente en los adolescentes varones que no estudian, existe una presencia mayor de conductas delictivas.

Concordando con investigaciones anteriores Pérez Correa (2013) recolecta datos acerca de la población carcelaria en el mundo y analiza que del total de esta población más del 95\% de prisioneros son hombres y el porcentaje restante son mujeres. El autor explica estos resultados a través de las teorías evolutivas que afirman que de acuerdo a diversos procesos los hombres desarrollaron la agresión como estrategia para escalar status, mantenerlo y con ello obtener acceso a relaciones sexuales.

Así mismo algunas teorías sociales como la de "Los roles de género" habla de una diferencia que abarca normas de comportamiento, actitudes, valores, creencias, tareas, etc. entre ambos sexos que según el Instituto Nacional de las Mujeres (INM, 2007) estos comportamientos y creencias surgen del espacio colectivo, en donde la herencia familiar y social a la que el individuo está sujeto sirven como medios de moldeamiento. 
Díaz-Guerrero (1994) señaló que en México el comportamiento de los niños y las niñas ha sido guiado para desempeñar actividades que van de acuerdo con su género a través de los agentes sociales más próximos a ellos y ellas que son los padres. En el caso de los niños se les orienta a jugar con soldaditos, pistolas, cascos, caballitos y espadas, por lo que es deducible que su comportamiento sea más agresivo en ellos, por el tipo de juego que desarrollan con estos objetos. Por el lado contrario, las niñas son orientadas a jugar con utensilios como son trastecitos, muñecos con apariencia de bebé, Barbies, entre otros, que están encaminados a desarrollar actividades ligadas a labores hogareñas y de cuidado personal y maternal, por lo que se espera que su comportamiento sea pasivo en comparación con el sexo opuesto.

La educación escolar también se encarga de promover estos estereotipos. Díaz-Guerrero (1994) señala que la educación en México enseña talleres orientados a prácticas en donde se refuerzan los comportamientos "típicos" masculinos o femeninos, si bien esto en el pasado era obligatorio, en la actualidad se convierte en un "derecho" a elegir, sin embargo la mayoría de niños y niñas eligen los talleres que están destinados para "hombres y mujeres", lo que habla de una internalización de las creencias y comportamientos sociales.

Si bien la presencia de conductas delictivas y antisociales se debe a diversos factores que juegan un espacio en la vida del individuo, es de suma importancia resaltar el amplio papel que juega la sociedad en los resultados obtenidos; la influencia que tiene esta en los roles de género, así como la interacción de los individuos en distintos espacios, se convierte en un elemento predisponente para la aparición de comportamientos delictivos y antisociales. Se enmarca de manera necesaria la modificación de diversas creencias y la creación de espacios donde los adolescentes puedan adquirir de una manera eficaz el conjunto de comportamientos que son deseables en ellos y que sean aceptados por la sociedad.

Queda también abierta la discusión a los sistemas actuales de educación que si bien parece funcionar como factores protectores de las conductas delictivas, no lo son del todo para evitar conductas antisociales. Muchos estudiantes consideran entre ellos, la existencia de conductas antisociales como parte de la convivencia cotidiana, ya que no les causa extrañeza que exista, pero sí ejerce un estado de incomodidad y conformismo (Romero \& Plata, 2015). Para el Observatorio sobre Violencia y Convivencia en la Escuela (como se citó en Benites 2012) si un clima es contante de inseguridad y temor para los estudiantes, contribuye de alguna manera, a la percepción de normalización y naturalización de la violencia, la agresión y el acoso, características fundamentales de las conductas antisociales. 


\section{References:}

1. Benites, L. (2012). La convivencia escolar: una estrategia de intervención en bullying. En L. Benites, J. Carozzo, V. Horna, L. Palomino, C. Salgado, C. Uribe \& L. Zapata Eds. (2012) Bullying y Convivencia en la Escuela. Aspectos conceptuales, aplicafivos y de invesfigación. Lima: .Impresiones \& Empastes.

2. Cava, M. J., Musitu, G., Buelga, S., y Murgui, S. (2010). The relationships of family and classroom environments with peer relational victimization: An analysis of their gender differences. The Spanish Journal of Psychology, 13(1), 156-165.

3. Centro de Investigación para el Desarrollo, [CIDAC]. (2015). \#Odisea2016 Historias de la transformación del sistema de justicia penal. México: CIDAC Y USAID. Recuperado de http://proyectojusticia.org/wpcontent/uploads/2015/11/ODISEA_2016_low.pdf

4. Cifuentes, J.J. \& Lodoño, N.H. (2011). Perfil cognitivo y psicopatológico asociados a la conducta antisocial. International Journal of Psychological Research. 4(1), 58-69.

5. Coon, D. \& Mitterer, J. (2011). Human Development. Psychology: A Journey. 4, 88-123.

6. D’alessandre, V. (2010). Adolescentes que no estudian ni trabajan en América Latina. Recuperado de http://www.siteal.com/198-23.

7. De la Peña, M. (2010). Conducta antisocial en adolescentes: factores de riesgo y de protección. (Tesis doctoral). Universidad Complutense de Madrid, Madrid.

8. Del Barrio, V. \& Roa, M. (2006). Factores de riesgo y protección en agresión infantil. Acción psicológica, 4(2), 39-65.

9. Díaz-Guerrero, R. (1994). Psicología del Mexicano; descubrimiento de la etnopscicología. México: Trillas.

10. Estevez, E. \& Emler, N. P. (2011). Assessing the links among adolescent and youth offender, antisocial behavior, victimization, drug use, and gender. International Journal of Clinical and Health Psychology, 11(2), 269-289.

11. Flores, D. (2010). La escuela como agente de socialización en los niños. (Tesina). Universidad de Cuenca, Cuenca.

12. Gaeta, M.L \& Galvanovskis, A. (2011). Propensión a Conductas Antisociales y Delictivas en Adolescentes Mexicanos. Psicología Iberoamericana, 19(2), 47-54.

13. Garaigordobil, M. (2005). Conducta Antisocial durante la Adolescencia correlatos socio-emocionales, predictores y diferencias de género. Psicología Conductual 3

14. Garaigordobil, M., Aliri, V., Martínez, V., Maganto, C., Bernaras, E. 
\& Jaureguizar, J. (2013). Conducta antisocial: conexión con emociones positivas y variables predictores. Apuntes de psicología, 31(2), 123-133.

15. Garcia, B. (2009). Metodología de la investigación. México: Manual Moderno

16. Gutiérrez, E., Torres, M., \& Fernanda M. (2011). Hannah Montana como modelo aspiracional de valores y consumo para niñas mexicanas: Un análisis cualitativo en el Noreste de México. Razón y palabra, (78). Recuperado de http://www.razonypalabra.org.mx/N/N78/07_GutierrezTorresForsbac h_M78.pdf.

17. Gutiérrez, J. P., Rivera-Dommarco, J., Shamah-Levy, T., Villalpando-Hernández, S., Franco, A., Cuevas-Nasu, L., ... \& Hernández-Ávila, M. (2012). Encuesta Nacional de Salud y Nutrición [ENSANUT] 2012. Resultados Nacionales. Cuernavaca: Instituto Nacional de Salud Pública (MX). Recuperado de http://ensanut.insp.mx/informes/ENSANUT2012ResultadosNacional es.pdf

18. Hein, A. \& Barrientos, G. (2004). Violencia y Delincuencia Juvenil: Comportamientos de riesgo autorreportados y factores asociados. Santiago: Fundación Paz Ciudadana.

19. Instituto Mexicano de la Juventud [IMJUVE]. (6 de Marzo de 2010). Instituto Mexicano de la Juventud. Recuperado el 15 de Noviembre de 2013, de IMJUVE: http://www.imjuventud.gob.mx/imgs/uploads/1._ENJ_2010_Hidalg o_VF_Mzo_6_MAC.pdf

20. Instituto Nacional de Estadística Geografía e Informática [INEGI]. (2014). Estadísticas a propósito del día internacional de la juventud (12 de agosto) Datos nacionales. Recuperado de http://www.inegi.org.mx/inegi/contenidos/espanol/prensa/contenidos/ estadisticas/2014/juventud0.pdf

21. Kazdin, A. E. \& Buela-Casal, G. (2006). Conducta antisocial. Evaluación, tratamiento y prevención en la infancia y adolescencia. Madrid: ediciones Pirámide.

22. López, C., Romero, A., \& López, R. (2016). Estilos de Apego en Niños de Preescolar: Una forma de Medición Válida, Confiable y Culturalmente Relevante. La Psicología Social en México, 16, 693697.

23. Marchiori, H. (2011). Psicología criminal. Mexico: Porrua, CA de C.V.

24. Martínez, L. C., Banqueri, V. M., \& Lozano, M. C. (2012). Consumo de drogas en adolescentes con conductas infractoras: análisis de 
variables psicosociales implicadas. Adolscencia Antisocial. Recuperado de http://adolescenciaantisocial.blogspot.mx/2012/03/consumo-dedrogas-en-adolescentes-con.html

25. Martínez, A.E. Inglés, C. Piqueras, J.A. \& Oblitas, L.A. (2010). Papel de la conducta prosocial y de las relaciones sociales en el bienestar psíquico y físico del adolescente. Avances en psicología latinoamericana. 28(1), 74-84.

26. Observatorio sobre Violencia y Convivencia en la Escuela. (2011). Impacto social de la violencia escolar. Informe preliminar. Lima: Peru. Recuperado de

27. Peres, M. (2008). Habilidades sociales en adolescentes institucionalizadas para el afrontamiento para su entorno inmediato. (Tesis Doctoral). Universidad de Granada. Granada.

28. Pérez, C., Gázquez, J., Mercader, I., Molero, M. \& García, M. (2011). Rendimiento académico y conductas antisociales y delictivas en alumnos de Educación Secundaria Obligatoria. International Journal of Psychology and Psychological Therapy, 11(3), 401-412.

29. Pérez Correa, C. (2013). Marcando al delincuente: estigmatización, castigo y cumplimiento del derecho. Revista mexicana de sociología, 75(2), 287-311.

30. Plata, J., Romero, A., Del Castillo, A., Domínguez, G. \& Martínez, A. (2015). Validación del inventario multidimensional de acoso psicológico en universitarios. Psicología Iberoamericana, 23(2), 3644.

Recuperado de http://oai.redalyc.org/articulo.oa?id=133944231005

31. Real Academia de la Lengua Española. (2001). Adolescencia. Recuperado de http://lema.rae.es/drae/srv/search?key=adolescencia

32. Rivas, Á. (2012). Habilidades Sociales y Relaciones Interpersonales. Fundación Gil Gayarre. Madrid, España. Recuperado de http://www.feaps.org/biblioteca/sexualidad_ydi/22_habilidades.pdf

33. Romero, A., \& Plata, J. (2015). Acoso escolar en universidades. Enseñanza e Investigación en Psicología, 20(3), 266274. Recuperado de http://oai.redalyc.org/articulo.oa?id=29242800003

34. Romero, A., Plata, J., Domínguez, G., Martínez, A., Del Castillo, A. \& Gil, F. (2014). Violencia y Acoso Escolar en Comunidades Universitarias: Comparación entre Universidades Públicas de Hidalgo y Tabasco. La Psicología Social en México, 15, 846-852.

35. Seisdedos, C. N., \& Sánchez, P. (2001). Cuestionario de conductas antisociales-delictivas A-D. México: El Manual Moderno.

36. SEP. (2010). Sistema Educativo de los Estados Unidos Mexicanos. 
Principales Cifras Ciclo Escolar 2010-2011. Recuperado de http://www.sep.gob.mx/work/models/sep1/Resource/1899/3/images/p rincipales_cifras_2010_2011.pdf

37. Fondo de las Naciones Unidas para la Infancia [UNICEF]. (2012). Observatorio de los Derechos de la Infancia y la Adolescencia en Uruguay 2012. Únete por la niñez. Recuperado de http://www.unicef.org/uruguay/spanish/observatorio-2012web20121030.pdf

38. Varela, J. (2011). Juventud, violencia y delincuencia desde una mirada de la prevención social del delito. Revista Observatorio de Juventud. (29), 39-54.

39. Vellado, F. (2014). Normas Jurídicas y normas de trato social. Recuperado de http://www.juridicas.unam.mx/publica/librev/rev/facdermx/cont/21/d tr/dtr3.pdf 